\title{
aSMA Osteoprogenitor Cells Contribute to the Increase in Osteoblast Numbers in Response to Mechanical Loading
}

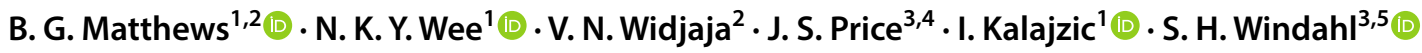

Received: 14 April 2019 / Accepted: 11 October 2019 / Published online: 31 October 2019

(c) The Author(s) 2019

\begin{abstract}
Bone is a dynamic tissue that site-specifically adapts to the load that it experiences. In response to increasing load, the cortical bone area is increased, mainly through enhanced periosteal bone formation. This increase in area is associated with an increase in the number of bone-forming osteoblasts; however, the origin of the cells involved remains unclear. Alpha-smooth muscle actin ( $\alpha \mathrm{SMA})$ is a marker of early osteoprogenitor cells in the periosteum, and we hypothesized that the new osteoblasts that are activated by loading could originate from $\alpha$ SMA-expressing cells. Therefore, we used an in vivo fate-mapping approach in an established axial loading model to investigate the role of $\alpha$ SMA-expressing cells in the load-induced increase in osteoblasts. Histomorphometric analysis was applied to measure the number of cells of different origin on the periosteal surface in the most load-responsive region of the mouse tibia. A single loading session failed to increase the number of periosteal $\alpha$ SMA-expressing cells and osteoblasts. However, in response to multiple episodes of loading, the caudal, but not the cranial, periosteal surface was lined with an increased number of osteoblasts originating from $\alpha$ SMA-expressing cells 5 days after the initial loading session. The proportion of osteoblasts derived from $\alpha$ SMA-labeled progenitors increased by $70 \%(p<0.05)$, and the proportion of $\alpha$ SMA-labeled cells that had differentiated into osteoblasts was doubled. We conclude that $\alpha$ SMA-expressing osteoprogenitors can differentiate and contribute to the increase in periosteal osteoblasts induced by mechanical loading in a site-specific manner.
\end{abstract}

Keywords Mouse $\cdot$ Axial loading $\cdot$ Lineage tracing $\cdot$ Osteoblasts $\cdot$ Osteoprogenitor

\section{Introduction}

Electronic supplementary material The online version of this article (https://doi.org/10.1007/s00223-019-00624-y) contains supplementary material, which is available to authorized users.

S. H. Windahl

Sara.windahl@ki.se

1 Department of Reconstructive Sciences, UConn Health, Farmington, CT, USA

2 Department of Molecular Medicine and Pathology, University of Auckland, Auckland, New Zealand

3 School of Veterinary Sciences, University of Bristol, Bristol, UK

4 Royal Agricultural University, Cirencester, UK

5 Division of Pathology, Department of Laboratory Medicine, Karolinska Institutet, Huddinge, Sweden
Mechanical loading as a result of load-bearing physical activity is a major regulator of bone mass and microarchitecture $[1,2]$. Load is sensed by bone cells as increased strain and results in modeling/remodeling processes that alter the bone architecture and form bones that are able to resist the habitual loads that are placed upon them. The increased bone formation is strain-dependent and site-specific; in response to axial loading of the mouse tibia, most bone is formed at the caudal periosteal site of the loaded bone [3-6]. Osteoblast numbers also increase following loading both in vivo $[7,8]$ and in vitro $[7,9]$. However, the origin of the cells that contribute to new osteoblast formation in this setting is not well defined. The aim of this study was to assess the contribution of $\alpha$ SMA-labeled progenitors to osteoblast formation in the periosteum following mechanical loading.

We have recently shown that bone lining cells represent a major source of osteoblasts during adulthood [10], and lining cells contribute to new osteoblasts in various anabolic 
settings including following treatment with sclerostin inhibitors or PTH $[11,12]$. Loading increases the number of periosteal osteoblasts in both male and female mice [7, 8]. However, the number of osteoblasts on an actively forming bone surface in response to load is approximately double that of the number of lining cells on a resting bone surface [8]. Since under most conditions, lining cells do not appear to proliferate following activation, osteoblasts must be recruited from cells other than lining cells in response to load $[11,12]$. It has been suggested that loading and unloading affect the number of proliferative osteoprogenitor cells in that loading increases [8] and unloading decreases [13, 14] their proliferation and recruitment. However, the identity of these osteoprogenitor cells is not well defined.

We have identified alpha-smooth muscle actin ( $\alpha$ SMA) as a marker of osteoprogenitor cells in bone [15-17]. These mesenchymal progenitors can differentiate into osteoblasts, adipocytes, and chondrocytes in vitro and in vivo and are located in perivascular niches, periosteum, and sutures. The fate of $\alpha$ SMA progenitor cells can be traced in vivo using tamoxifen-inducible $\alpha$ SMACreERT2 combined with a reporter. The $\alpha$ SMA-labeled cells in the periosteum and other tissues do not express markers of mature osteoblasts including transgenic Col2.3GFP a short period after labeling $[15,17,18]$. Using this model, we have demonstrated differentiation of tissue-resident $\alpha$ SMA-labeled cells in various settings. Specifically, $\alpha$ SMA-labeled cells contribute to osteoblasts and osteocytes during adolescent growth, cementoblasts, and periodontal ligament cells in the periodontium during growth and following injury, and odontoblasts following reparative dentinogenesis $[15,18,19]$. In the periosteum, $\alpha$ SMA-labeled cells are present in adulthood and contribute to osteoblasts, chondrocytes, and fibroblasts following fracture $[15,17]$.

In the present study, we have combined lineage tracing of $\alpha$ SMA-labeled cells, with a well-established marker of mature osteoblasts, Col2.3GFP [20], to track to origin of osteoblasts in the periosteum following an anabolic loading stimulus.

\section{Materials and Methods}

\section{Mice}

Procedures were approved by the UConn Health Institutional Animal Care and Use Committee and performed in an AAALAC accredited facility. Mice were housed in ventilated cages with a 12-h light cycle and temperature of $22^{\circ} \mathrm{C}$. Water and irradiated rodent chow (Teklad 2918, Invigo, Indianapolis, IN) was provided ad libitum. Male $\alpha$ SMACreER/ Ai9/2.3GFP mice in a C57B1/6J background were used for all studies and have been previously described [15, 20, 21].

\section{Mechanical Strain Measurements During Dynamic Axial Loading}

The strain:load relationship in the tibiae of 4-month-old male C57BL/6 mice was performed as previously described [22, 23]. In short, strains were measured using a single element strain gauge (EA-06-015DJ-120, Vishay Measurement Group, PA, USA) at the medial aspect of the tibia at approximately $37 \%$ of its length from the proximal end across a range of peak compressive loads between 6 and $18 \mathrm{~N}$ (Fig. 1a). From the data, a peak strain corresponding to $2400 \mu \varepsilon$ was reached at $11.4-11.6 \mathrm{~N}$.

\section{In Vivo Axial Loading of the Tibia}

At 16-20 weeks of age, male mice were anaesthetized using isoflurane and their right tibiae were axially loaded. This involved positioning the tibia within the loading cups, securing in place, and applying 11.4 or $11.6 \mathrm{~N}$ (for the single and multiple episodes of loading, respectively) force at $1 \mathrm{~Hz}$ for 40 cycles, with a 10-s rest in between cycles using a 3100 ElectroForce ${ }^{\circledR}$ Test Instrument (TA Instruments, DE, USA) $[22,23]$. The left tibia from the same mouse was used as a non-loaded control as previously validated [4, 5, 24].

\section{Experimental design}

In order to stimulate new bone formation, loading is generally applied over a number of days; however, responses such as reduction in Sclerostin expression are evident $24 \mathrm{~h}$ after a single round of loading [7] and it has been suggested that a single loading is enough to promote endosteal and periosteal osteoblast proliferation in rats and roosters $[8,25]$. We, therefore, divided the mice in two main groups receiving either a single session or multiple episodes of loading.

\section{Single loading}

Tamoxifen (75ug/g of body weight; T5648; Sigma) was administered on days -3 and -2 , then tibial loading performed on day 0 . EdU (5-ethynyl-2'-deoxyuridine, $100 \mu \mathrm{l}$ of $1 \mathrm{mg} / \mathrm{ml}$ solution) was administered twice daily to label proliferating cells. The animals were euthanized on days 3 $(N=6)$ and $7(N=5$, Fig. $1 b)$.

\section{Multiple Episodes of Loading}

Two days prior to the study, male mice were pair-housed to limit natural physical activity within the cage yet maintain social interactions. Tamoxifen was administered on days $-2,0$, and 2 , and tibial loading was performed on days 
Fig. 1 The load strain relationship and experimental outlines. a The load strain relationship shows that $2400 \mu \varepsilon$ was reached around $11.5 \mathrm{~N}$. b Experimental design for the single loading experiment. EdU was injected twice daily. c Experimental design for the multiple episodes of loading experiment. d Tibial cross section indicating regions of the periosteum analyzedthe caudal region is indicated in red, and the cranial region in yellow.
A

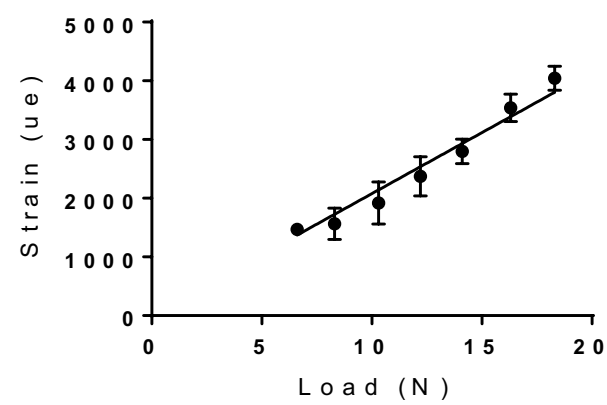

B

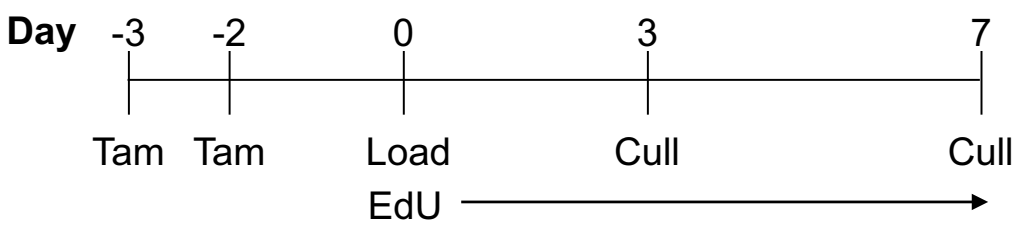

C

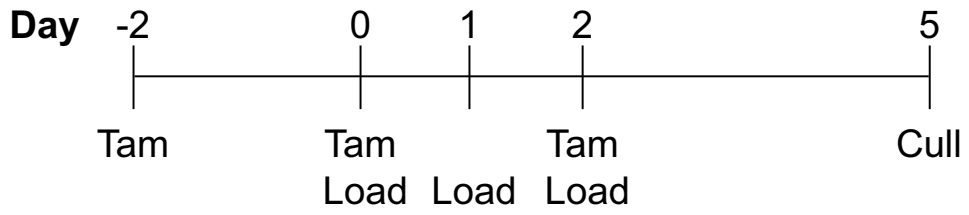

D

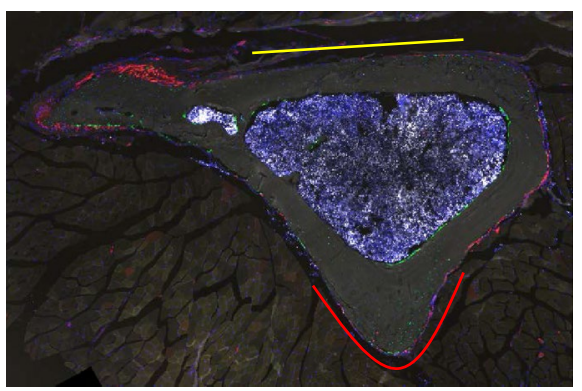

0,1 , and 2 . The animals were euthanized on day $5(N=8)$ (Fig. 1c). Another cohort of animals were given tamoxifen $(N=4)$ or vehicle (corn oil $N=6)$ without undergoing the loading regimen.

\section{Tissue Collection, Sectioning, and Imaging}

Mice were administered ketamine/xylazine cocktail and intracardiac perfusion was performed with PBS followed by $4 \%$ paraformaldehyde and fixed for 2 days. Tibiae were decalcified using 14\% EDTA for 2-3 weeks, then transferred to $30 \%$ sucrose at $4{ }^{\circ} \mathrm{C}$, prior to cryo-embedding with Cryomatrix (Thermo Fisher Scientific, Waltham, MA, USA). Bones were embedded to facilitate obtaining tibial cross sections, located approximately $37 \%$ from the proximal end of the tibia. Tissues were cut at $7 \mu \mathrm{m}$ thickness on Cryotape (Cryofilm 2C, Section Lab, Japan) and cross-linked using Norland Optical Adhesive 61 (Norland Optical, Cranbury, $\mathrm{NJ}$ ) onto glass slides. Sections were subsequently stained with Click-iT EdU Plus Imaging Kit, according to the manufacturer's instructions (Alexa Fluor 647; Invitrogen). Slides were coverslipped in 50\% glycerol with DAPI (1:5000, D1306; Molecular Probes). Slides were imaged using an Axioscan Z1 (Zeiss) with standardized settings maintained throughout each experiment.

\section{Image Analysis}

For the image analysis, 3-6 sections of each tibia were analyzed. We targeted two regions for analysis, the caudal 
and cranial surfaces, which sense compressive and tensile strain, respectively, during loading (Fig. 1d). In response to axial mechanical loading, bone formation is induced at both these sites, but the response is stronger at the caudal than the cranial site $[3,4,26]$. For the single loading study, nuclei were counted manually in the ROIs using Zen software (Zeiss), followed by determination of the number of cells expressing one or more fluorescent labels. For the multiple episodes of loading study, analysis was performed using ImageJ2 software (NIH, Bethesda, MD, USA). The DAPI channel underwent background subtraction followed by thresholding using the Li method and segregation by the watershed function. Particle analysis was performed and nuclei were counted based on size $\left(10-150 \mu^{2}\right)$ and circularity $(>0.5)$ within the ROIs, and signals in the red and green channels measured. Thresholds were determined manually and applied in Excel. The length of the bone surface analyzed was also measured. Osteoblast counts were determined by dividing the number of GFP+ cells by the length of the surface evaluated, $\alpha$ SMA numbers were calculated in a similar manner using the number of tdTomato+ cells. For $\alpha$ SMA differentiation, the number of dual positive GFP+ tdTomato+ cells was divided by total tdTomato+ cells. For osteoblasts derived from $\alpha \mathrm{SMA}+$ cells, the number of dual positive cells was divided by total GFP+ cells.

\section{Statistical Analyses}

Statistical analysis was performed in GraphPad Prism 7. Normality tests suggested that the datasets were not normally distributed so Wilcoxon matched-pairs signed rank tests were performed. The proportion of osteoblasts derived from $\alpha \mathrm{SMA}+$ cells in the caudal region was considered the primary outcome, where $p<0.05$ is statistically significant. For other comparisons, a Bonferonni correction was applied so the adjusted significance threshold was $p<0.00714$. Full $\mathrm{p}$ value results for the multiple loading experiment are shown in Table 1.

Table $1 P$ values associate with Wilcoxon matched-pairs signed rank test for multiple loading parameters (Fig. 4)

\begin{tabular}{lllll}
\hline & N.oSMA+/BS & N.Ob/BS & $\begin{array}{l}\alpha \text { SMA+ } \\
\text { differentia- } \\
\text { tion }\end{array}$ & $\begin{array}{l}\text { Ob from } \\
\alpha \text { SMA+ } \\
\text { cells }\end{array}$ \\
\hline Caudal L vs R & 0.1953 & 0.1484 & 0.0391 & $\mathbf{0 . 0 1 5 6 *}$ \\
Cranial L vs R & 0.6406 & 0.0078 & 0.3125 & 0.6719 \\
\hline
\end{tabular}

* Primary outcome, therefore $p<0.05$ is statistically significant. Significant $p$ value for other parameters following Bonferonni correction is $p<0.00714$

\section{Results}

The effect of tamoxifen on osteoblast numbers was tested following three injections of tamoxifen. We did not find any difference in the number of osteoblasts per bone surface in either the cranial or caudal sites (Supplemental Fig. 1).

\section{A Single Session of Intermittent Loading Does Not Enhance the Number of Periosteal aSMA+ Cells or Osteoblasts}

We initially evaluated the effect of a single round of axial loading on the number of Col2.3GFP+ osteoblasts, $\alpha$ SMAlabeled cells, and proliferating cells. We were unable to detect any difference in parameters associated with a bone formation response to loading either 3 or 7 days following loading. Specifically, there was no increase in the number of $\alpha$ SMA-labeled cells or GFP+ osteoblasts in the loaded limb (Fig. 2a, b), nor was there any change in the contribution of $\alpha$ SMA-labeled cells to osteoblasts with response to loading or in relation to the time since loading and labeling (Fig. 2c, d). While we observed EdU incorporation into a subset of periosteal cells, including both $\alpha$ SMA-labeled cells and osteoblasts, at the time points investigated loading did not significantly change proliferation in the regions analyzed (Figs. 2e, f and 3).

\section{Multiple Episodes of Axial Loading of the Tibia Enhances the Number of Differentiating aSMA-Positive Cells and Osteoblasts Derived from aSMA+ Cells at the Caudal Periosteal Site}

In order to enhance the bone formation response to mechanical loading, we loaded tibiae on three consecutive days. Five days after initiating multiple episodes of loading, we did not detect any difference in the nuclei/ $\mu \mathrm{m}$ (data not shown) or in the number of $\alpha \mathrm{SMA}+$ cells at either the caudal or cranial sites (Fig. 4a, Table 1). The number of GFP+ osteoblasts increased by $58 \%$ at the cranial site. At the caudal site, the change was not statistically significant, although $6 / 8$ animals showed increased osteoblast numbers, representing more than doubling of the osteoblast numbers on average in the caudal site of the loaded limb (Fig. 4b, e-1). However, differentiation of $\alpha$ SMA-labeled progenitor cells into osteoblasts was significantly enhanced in the caudal region. We saw both a 70\% increase in the proportion of osteoblasts derived from $\alpha$ SMA-labeled progenitors (Fig. $4 \mathrm{c}$ ) and a doubling in the proportion of $\alpha$ SMA-labeled cells that had differentiated into osteoblasts at the caudal site (Fig. 4d). Contribution of 
Fig. 2 A single session of intermittent loading does not enhance the number of periosteal $\alpha \mathrm{SMA}+$ cells or osteoblasts $(\mathrm{Ob})$. a $\alpha \mathrm{SMA}$ labeled cell numbers and $\mathbf{b}$ GFP+ osteoblast numbers per bone surface in the periosteum in the indicated regions. c The percentage of osteoblasts derived from $\alpha$ SMA-expressing cells and $\mathbf{d}$ the percentage of $\alpha \mathrm{SMA}+$ cells that differentiated into osteoblasts. e The percentage of $\alpha$ SMA-labeled cells that proliferated following loading and f GFP+ osteoblasts that proliferated following loading. Bars show mean \pm SEM, $n=6$ for day $3, n=5$ for day $7 . * p<0.05$
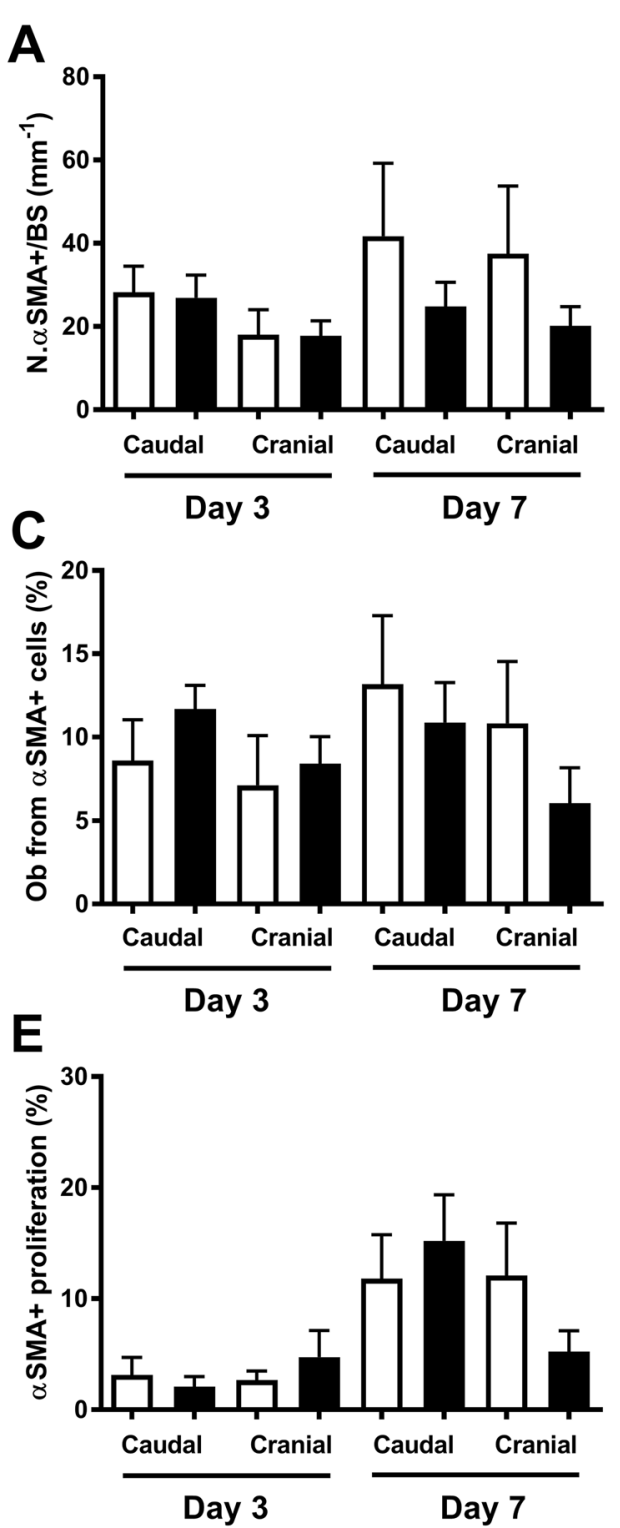
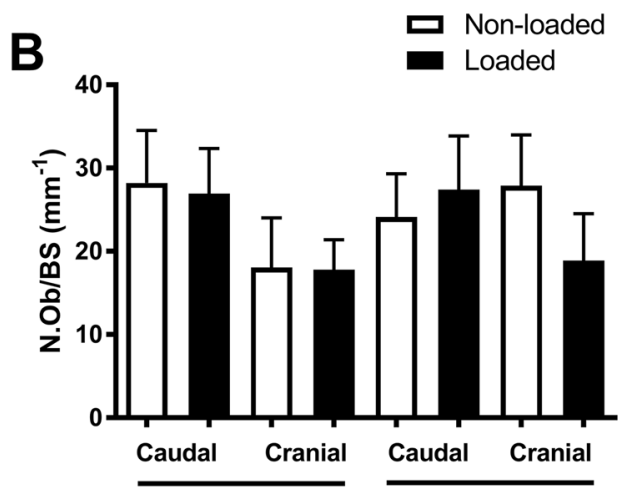

Day 3

Day 7

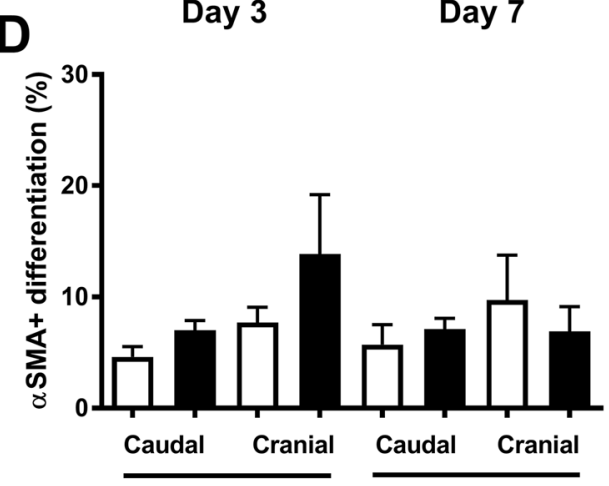

$\mathbf{F}$

Day 3

Day 7

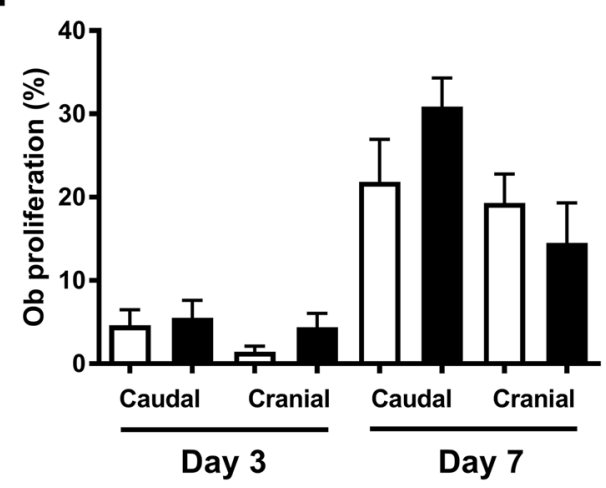

$\alpha$ SMA-labeled cells to osteoblasts did not increase in the cranial region.

\section{Discussion}

Mechanical loading causes a rapid anabolic response in specific regions of the periosteum [3-6]. However, the origin of the cells that contribute to new osteoblast formation in this setting is not well defined. In this study, we assessed the contribution of $\alpha$ SMA-labeled progenitors to osteoblast formation following mechanical loading. We have previously demonstrated that $\alpha$ SMACreERT2 labels a population of progenitors in the periosteum that make a major contribution to both osteoblasts and chondrocytes within the fracture callus, but does not initially identify osteoblasts $[15,17]$.
The bone anabolic response to loading is site-specific, where most bone formation is seen at the periosteal caudal site $[3,26]$. Therefore, we investigated the number of osteoblasts and their origin at the periosteal caudal site and compared it to the cranial surface where less bone is formed in response to load. We were unable to see evidence of enhanced osteoblast proliferation or differentiation of osteoprogenitors into osteoblasts with a single episode of loading. This is in contrast with previous work in rats and roosters where a single round of loading promoted an increase in osteoblast numbers and bone formation 3-5 days after loading $[8,25]$. The apparent discrepancy could be related to differences in sex, strain applied, or species differences. Although the study in rats did not report the strains applied, the study in roosters applied strains of 2700-3000 $\mu$ strain [25], which is higher than the strains used in this study. 


\section{Non-loaded}
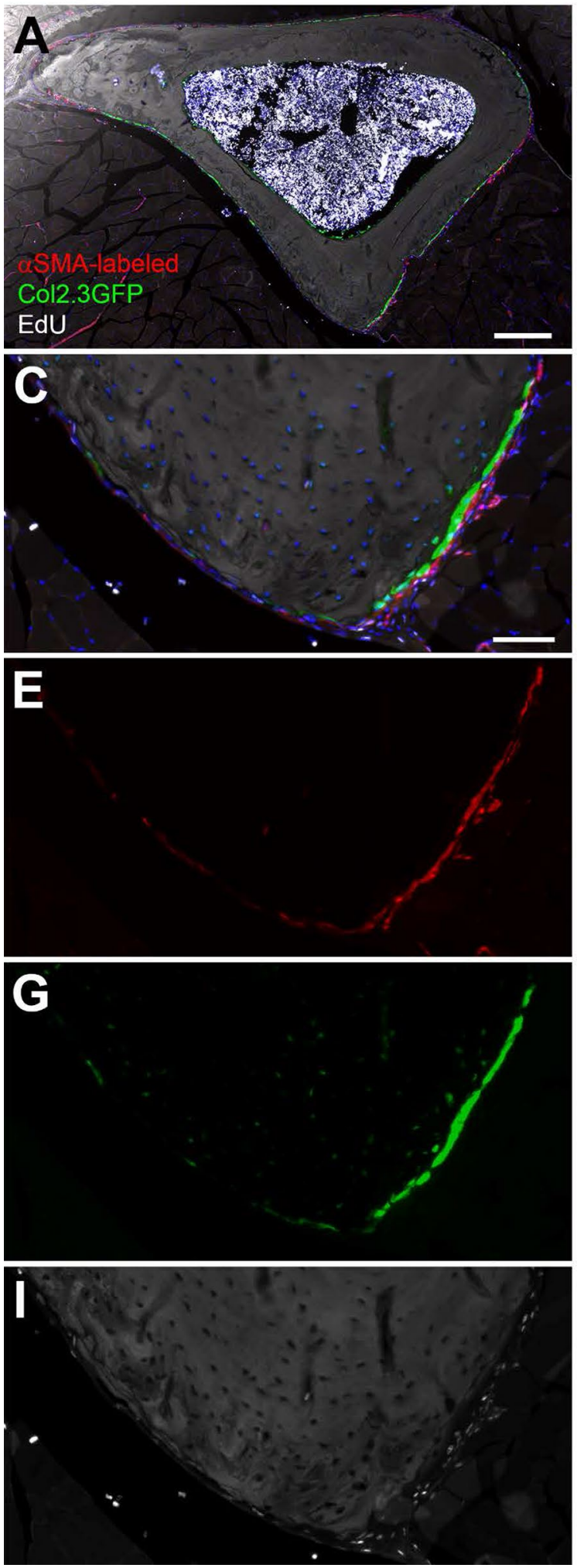

Fig. 3 Representative images of non-loaded (a, c, e, g, and i) and loaded ( $\mathbf{b}, \mathbf{d}, \mathbf{f}, \mathbf{h}$, and $\mathbf{j})$ bones. The caudal region is magnified in $\mathbf{c}-\mathbf{j}$. Overview of the tibia cross section (a and $\mathbf{b}), \alpha \mathrm{SMA}+$ cells in red (e and $\mathbf{f}), \mathrm{Col} 2.3 \mathrm{GFP}+$ osteoblasts in green $(\mathbf{g}$ and $\mathbf{h})$, and EdU+ cells in

\section{Loaded}
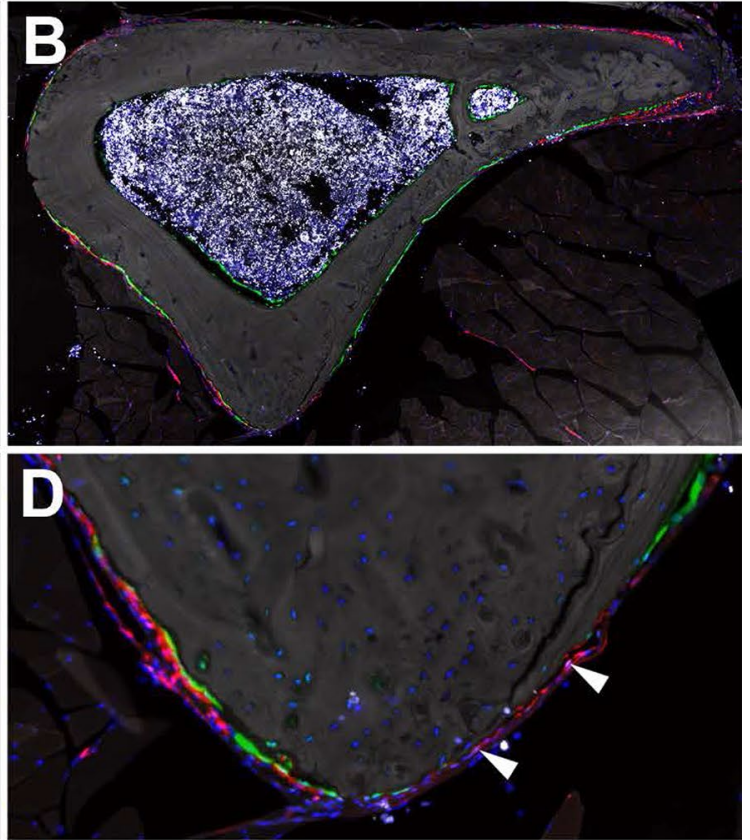

$\mathbf{F}$

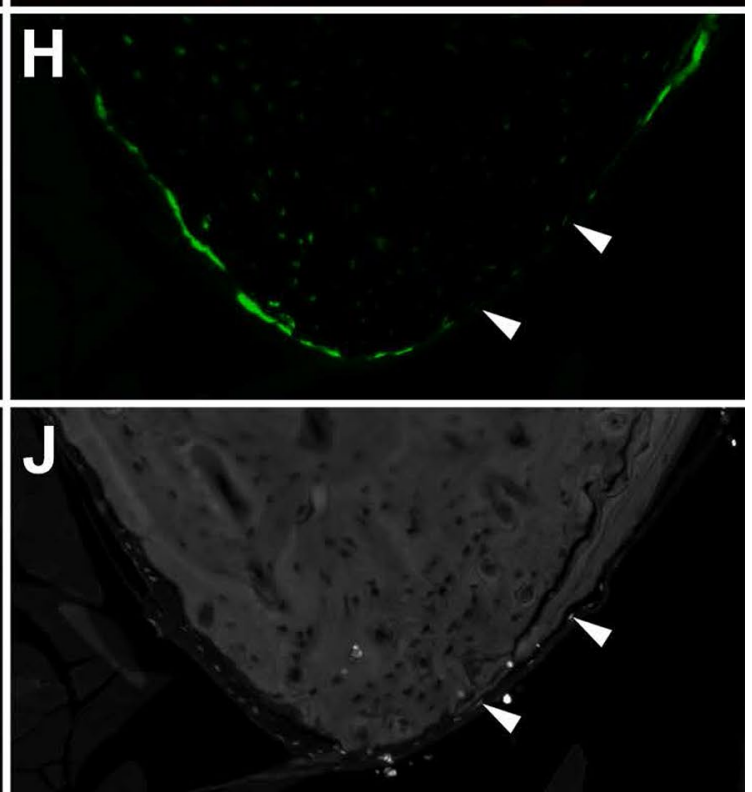

white (i and $\mathbf{j})$. DAPI staining is shown in blue. Scale bars represent $200 \mu \mathrm{m}(\mathbf{a}, \mathbf{b})$ or $50 \mu \mathrm{m}(\mathbf{c}, \mathbf{j})$. Arrowheads indicate $\alpha \mathrm{SMA}+\mathrm{EdU}+$ cells in the caudal region 

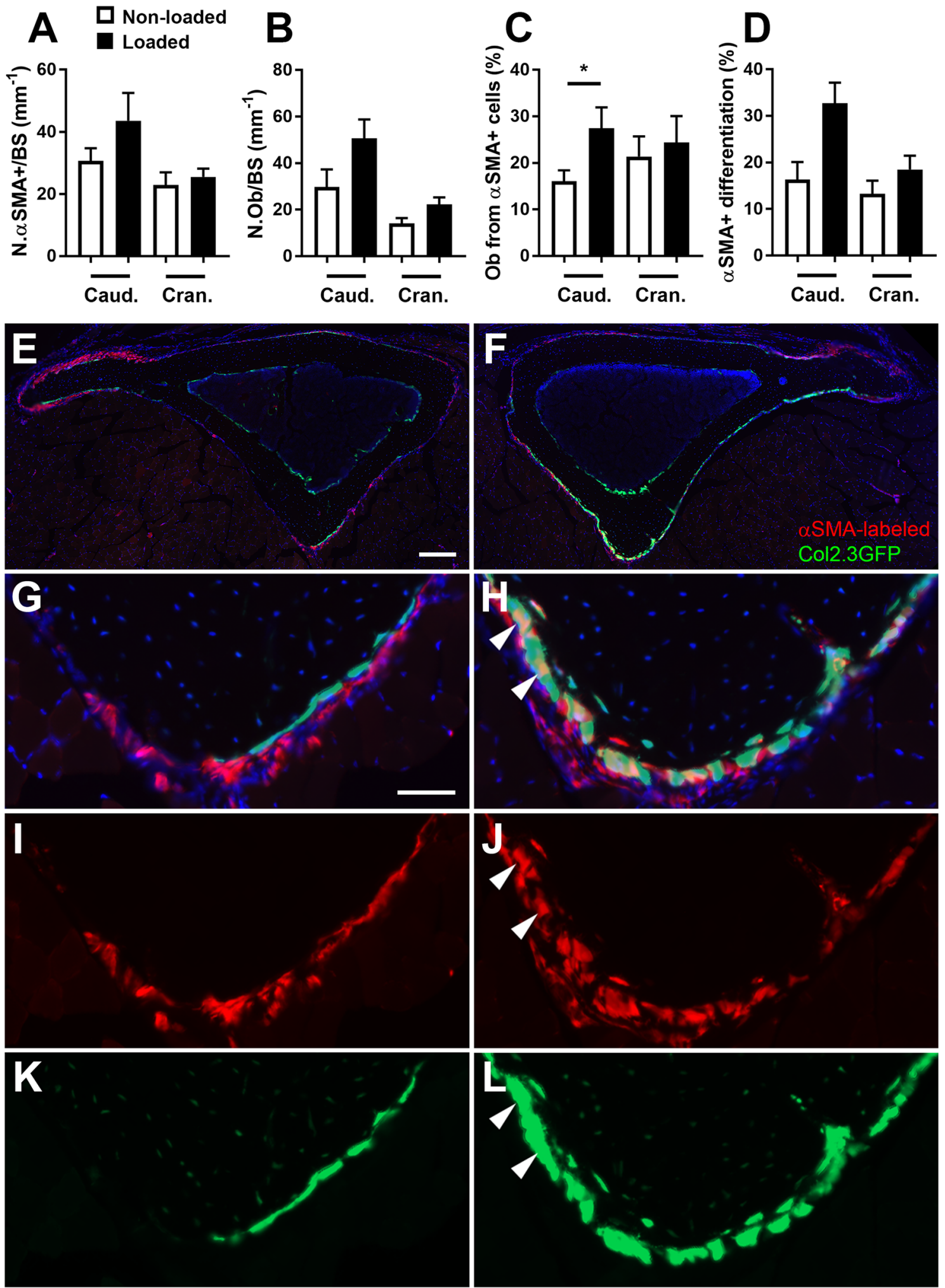

Fig. 4 Axial loading of the tibia enhances differentiation of periosteal $\alpha \mathrm{SMA}+$ cells into osteoblasts $(\mathrm{Ob})$ after multiple episodes of loading. a $\alpha$ SMA-labeled cell numbers and b GFP+ osteoblast numbers per bone surface in the periosteum in the indicated regions. c The percentage of osteoblasts derived from $\alpha \mathrm{SMA}$-expressing cells and $\mathbf{d}$ the percentage of $\alpha \mathrm{SMA}+$ cells that differentiated into osteoblasts. Rep- resentative images of non-loaded $(\mathbf{e}, \mathbf{g}, \mathbf{i}$, and $\mathbf{k})$ and loaded $(\mathbf{f}, \mathbf{h}, \mathbf{j}$, and $\mathbf{l})$ bones are shown with the caudal region magnified in $\mathbf{g}-\mathbf{l}$. DAPI staining is depicted in blue. Bars show mean \pm SEM, $n=8$. * statistically significant (see methods for $\mathrm{p}$ value thresholds). Scale bars represent $200 \mu \mathrm{m}(\mathbf{e}, \mathbf{f})$ or $50 \mu \mathrm{m}(\mathbf{g}-\mathbf{l})$. Arrowheads indicate selected $\alpha \mathrm{SMA}+\mathrm{Col} 2.3 \mathrm{GFP}+$ cells . 
In contrast to single loading, multiple episodes of loading enhanced the osteoblast number in the periosteum in 6/8 animals. This is in line with a previous study showing that the periosteal bone-forming surface increases in a dose-dependent manner with increasing number of loading sessions [27]. The authors of that study proposed that a quantum of bone cells is activated in response to each loading event, whereas the strain magnitude dictates the size or microstructural organization of each quantum of bone cells.

Following multiple episodes of loading, while osteoblast number did not increase in all samples in the caudal region where most bone is formed in response to load $[3,26]$, we consistently saw increased contribution of $\alpha \mathrm{SMA}+$ progenitors to the osteoblast pool, indicating that loading promoted increased differentiation of these cells. This was not seen in the cranial region where less load response is seen. The mechanisms whereby load stimulates differentiation of $\alpha \mathrm{SMA}+$ progenitors are unknown, although they are likely to involve osteocytes sensing the increasing strain in the bone and then communicating with the periosteal $\alpha \mathrm{SMA}+$ progenitors via signaling molecule/s. We have demonstrated previously that $\alpha$ SMA labels periosteal-resident cells in adult mice $[15,17]$. These are a mixed population of cells, but a subset expresses mesenchymal stem and progenitor markers such as Sca1. We cannot completely exclude contribution of skeletal muscle-derived cells as $\alpha$ SMA does label both myogenic and mesenchymal populations in the muscle [28]. However, studies of fracture healing indicate that muscle cells can act as chondroprogenitors, but only contribute to osteoblasts within the fracture callus when the periosteum is extensively damaged or removed [29, 30]. On this basis, we believe it is highly unlikely that muscle-resident cells are contributing to osteoblast formation following loading. Studies using bone-restricted markers like Osterix during loading appear to confirm this [31].

It is difficult to estimate the exact contribution of the $\alpha \mathrm{SMA}+$ progenitor population to loading-based bone formation for a number of reasons. Firstly, most of the surfaces analyzed, even those from non-loaded bones, had Col2.3GFP+ osteoblasts present. The lifespan of osteoblasts in vivo is difficult to define, but recent studies suggest around 2 weeks is a reasonable estimate in adult mice [10, 32]. Therefore, some of the osteoblasts on the surfaces analyzed were likely present prior to tamoxifen delivery, meaning they could not have been $\alpha$ SMA-labeled. Assuming the non-loaded leg represents the number of 'old' osteoblasts, we estimate that an average of $52 \%$ of 'new' osteoblasts is derived from $\alpha \mathrm{SMA}+$ progenitors. However, given there are osteoblasts derived from $\alpha$ SMA-progenitors in the unloaded limbs as well, new osteoblast formation was clearly also occurring on these surfaces under both loading regimens. In addition, the efficiency of the inducible Cre recombination is rarely $100 \%$. Indeed, $\alpha$ SMACreER labels about $50 \%$ of $\alpha$ SMA-expressing cells when analyzed in vitro (BGM, IK in preparation). Thus, it is difficult to estimate exactly what proportion of the newly recruited osteoblasts are originating from $\alpha \mathrm{SMA}$ osteoprogenitors, but our numbers suggest that there is another source of osteoblasts outside the $\alpha \mathrm{SMA}$ lineage. A recent study showed that genetic ablation of Prx1CreER cells, which likely represent a similar, or at least overlapping subset of periosteal progenitors, significantly reduced bone formation rate in response to ulnar loading [33]. Although reduced, the anabolic response to loading was not completely lost, despite evidence that ablation was close to $100 \%$ effective, supporting the idea that a non-progenitor population such as bone lining cells also contributes to the loading response. It was also recently reported that osterix (Osx) lineage cells identified in adulthood using OsxCreER contribute to most or all of the bone surface cells associated with mineralization within the days following loading in a similar model [31]. Osx is expressed throughout the osteoblast lineage including in progenitors, osteoblasts, and bone lining cells, and we have previously demonstrated Osx expression in $\alpha \mathrm{SMA}+$ periosteal cells $[10,17]$. These results are therefore consistent with our conclusion that $\alpha \mathrm{SMA}+$ progenitors contribute to some osteoblasts while others likely arise from more differentiated cells within the lineage that no longer express $\alpha$ SMA.

Our study has some limitations in that we were not able to show enhanced osteoblast proliferation or osteoprogenitor differentiation into osteoblasts with a single round of loading. It is possible that if we had used a higher strain magnitude, we may have been able to detect a significant increase in osteoblast differentiation and/or number. However, a higher strain may not reflect the strains that are normally placed upon the skeleton. Another limitation of this study is that we induced the expression of the reporter transgene by tamoxifen. Tamoxifen is a selective estrogen receptor modulator known to independently enhance bone formation and to modify the loading response at concentrations at or below what is used for most lineage tracing studies [24]. However, in agreement with Zannit and Silva [31], we could not detect any statistically significant difference in osteoblast number per bone surface when comparing vehicle and tamoxifentreated mice. While some osteoblasts may have been present prior to tamoxifen or loading, our results indicate that another population in addition to $\alpha$ SMA-labeled cells contributes to osteoblasts in this scenario.

In conclusion, this study has demonstrated that mechanical loading increases the differentiation of osteoblasts from $\alpha$ SMA-labeled progenitors at specific sites on the periosteal surface.

Acknowledgements Open access funding provided by Karolinska Institute. This work was supported by European Union's Horizon 2020 research and innovation programme under the Marie Skłodowska-Curie 
grant agreement No. 657178 and the Swedish Research Council (20138252) to SHW, NIH/NIAMS grant R01AR055607 to IK, Connecticut Stem Cell grant 14-SCA-UCHC-02, and Health Research Council of New Zealand Sir Charles Hercus Fellowship to BGM. We thank Dr Nicholas Knowlton, University of Auckland for assistance with statistical analysis.

\section{Compliance with Ethical Standards}

Conflict of interest The authors have no conflicts of interest to declare.

Human and Animal Rights and Informed Consent All applicable international, national, and/or institutional guidelines for the care and use of animals were followed. All procedures performed in the studies involving animals were approved by and in accordance with the ethical standards of the UConn Health Institutional Animal Care and Use Committee and performed in an AAALAC accredited facility.

Open Access This article is distributed under the terms of the Creative Commons Attribution 4.0 International License (http://creativeco mmons.org/licenses/by/4.0/), which permits unrestricted use, distribution, and reproduction in any medium, provided you give appropriate credit to the original author(s) and the source, provide a link to the Creative Commons license, and indicate if changes were made.

\section{References}

1. Frost HM (1987) Bone "mass" and the "mechanostat": a proposal. Anat Rec 219:1-9

2. Wolff J (2010) The classic: on the inner architecture of bones and its importance for bone growth. 1870. Clin Orthop Relat Res 468:1056-1065

3. Bergstrom I, Kerns JG, Tornqvist AE, Perdikouri C, Mathavan N, Koskela A, Henriksson HB, Tuukkanen J, Andersson G, Isaksson H, Goodship AE, Windahl SH (2017) Compressive loading of the murine tibia reveals site-specific micro-scale differences in adaptation and maturation rates of bone. Osteoporos Int 28:1121-1131

4. De Souza RL, Matsuura M, Eckstein F, Rawlinson SC, Lanyon LE, Pitsillides AA (2005) Non-invasive axial loading of mouse tibiae increases cortical bone formation and modifies trabecular organization: a new model to study cortical and cancellous compartments in a single loaded element. Bone 37:810-818

5. Galea GL, Hannuna S, Meakin LB, Delisser PJ, Lanyon LE, Price JS (2015) Quantification of alterations in cortical bone geometry using site specificity software in mouse models of aging and the responses to ovariectomy and altered loading. Front Endocrinol (Lausanne) 6:52

6. Turner CH, Forwood MR, Rho JY, Yoshikawa T (1994) Mechanical loading thresholds for lamellar and woven bone formation. $\mathrm{J}$ Bone Miner Res 9:87-97

7. Meakin LB, Galea GL, Sugiyama T, Lanyon LE, Price JS (2014) Age-related impairment of bones' adaptive response to loading in mice is associated with sex-related deficiencies in osteoblasts but no change in osteocytes. J Bone Miner Res 29:1859-1871

8. Turner CH, Owan I, Alvey T, Hulman J, Hock JM (1998) Recruitment and proliferative responses of osteoblasts after mechanical loading in vivo determined using sustained-release bromodeoxyuridine. Bone 22:463-469

9. Lee K, Jessop H, Suswillo R, Zaman G, Lanyon L (2003) Endocrinology: bone adaptation requires oestrogen receptor-alpha. Nature 424:389
10. Matic I, Matthews BG, Wang X, Dyment NA, Worthley DL, Rowe DW, Grcevic D, Kalajzic I (2016) Quiescent bone lining cells are a major source of osteoblasts during adulthood. Stem Cells 34:2930-2942

11. Kim SW, Lu Y, Williams EA, Lai F, Lee JY, Enishi T, Balani DH, Ominsky MS, Ke HZ, Kronenberg HM, Wein MN (2017) Sclerostin antibody administration converts bone lining cells into active osteoblasts. J Bone Miner Res 32:892-901

12. Kim SW, Pajevic PD, Selig M, Barry KJ, Yang JY, Shin CS, Baek WY, Kim JE, Kronenberg HM (2012) Intermittent parathyroid hormone administration converts quiescent lining cells to active osteoblasts. J Bone Miner Res 27:2075-2084

13. Keila S, Pitaru S, Grosskopf A, Weinreb M (1994) Bone marrow from mechanically unloaded rat bones expresses reduced osteogenic capacity in vitro. J Bone Miner Res 9:321-327

14. Zhang R, Supowit SC, Klein GL, Lu Z, Christensen MD, Lozano R, Simmons DJ (1995) Rat tail suspension reduces messenger RNA level for growth factors and osteopontin and decreases the osteoblastic differentiation of bone marrow stromal cells. J Bone Miner Res 10:415-423

15. Grcevic D, Pejda S, Matthews BG, Repic D, Wang L, Li H, Kronenberg MS, Jiang X, Maye P, Adams DJ, Rowe DW, Aguila HL, Kalajzic I (2012) In vivo fate mapping identifies mesenchymal progenitor cells. Stem Cells 30:187-196

16. Kalajzic Z, Li H, Wang LP, Jiang X, Lamothe K, Adams DJ, Aguila HL, Rowe DW, Kalajzic I (2008) Use of an alphasmooth muscle actin GFP reporter to identify an osteoprogenitor population. Bone 43:501-510

17. Matthews BG, Grcevic D, Wang L, Hagiwara Y, Roguljic H, Joshi P, Shin DG, Adams DJ, Kalajzic I (2014) Analysis of alphaSMA-labeled progenitor cell commitment identifies notch signaling as an important pathway in fracture healing. J Bone Miner Res 29:1283-1294

18. Roguljic H, Matthews BG, Yang W, Cvija H, Mina M, Kalajzic I (2013) In vivo identification of periodontal progenitor cells. J Dent Res 92:709-715

19. Vidovic I, Banerjee A, Fatahi R, Matthews BG, Dyment NA, Kalajzic I, Mina M (2017) alphaSMA-expressing perivascular cells represent dental pulp progenitors in vivo. J Dent Res 96:323-330

20. Kalajzic I, Kalajzic Z, Kaliterna M, Gronowicz G, Clark SH, Lichtler AC, Rowe D (2002) Use of type I collagen green fluorescent protein transgenes to identify subpopulations of cells at different stages of the osteoblast lineage. J Bone Miner Res $17: 15-25$

21. Madisen L, Zwingman TA, Sunkin SM, Oh SW, Zariwala HA, Gu H, Ng LL, Palmiter RD, Hawrylycz MJ, Jones AR, Lein ES, Zeng H (2010) A robust and high-throughput Cre reporting and characterization system for the whole mouse brain. Nat Neurosci 13:133-140

22. Todd H, Galea GL, Meakin LB, Delisser PJ, Lanyon LE, Windahl SH, Price JS (2015) Wnt16 Is associated with age-related bone loss and estrogen withdrawal in murine bone. PLoS ONE 10:e0140260

23. Windahl SH, Saxon L, Borjesson AE, Lagerquist MK, Frenkel B, Henning P, Lerner UH, Galea GL, Meakin LB, Engdahl C, Sjogren K, Antal MC, Krust A, Chambon P, Lanyon LE, Price JS, Ohlsson C (2013) Estrogen receptor-alpha is required for the osteogenic response to mechanical loading in a ligand-independent manner involving its activation function 1 but not 2 . J Bone Miner Res 28:291-301

24. Sugiyama T, Galea GL, Lanyon LE, Price JS (2010) Mechanical loading-related bone gain is enhanced by tamoxifen but unaffected by fulvestrant in female mice. Endocrinology 151:5582-5590

25. Pead MJ, Skerry TM, Lanyon LE (1988) Direct transformation from quiescence to bone formation in the adult periosteum 
following a single brief period of bone loading. J Bone Miner Res 3:647-656

26. Moustafa A, Sugiyama T, Prasad J, Zaman G, Gross TS, Lanyon LE, Price JS (2012) Mechanical loading-related changes in osteocyte sclerostin expression in mice are more closely associated with the subsequent osteogenic response than the peak strains engendered. Osteoporos Int 23:1225-1234

27. Forwood MR, Turner CH (1994) The response of rat tibiae to incremental bouts of mechanical loading: a quantum concept for bone formation. Bone 15:603-609

28. Matthews BG, Torreggiani E, Roeder E, Matic I, Grcevic D, Kalajzic I (2016) Osteogenic potential of alpha smooth muscle actin expressing muscle resident progenitor cells. Bone 84:69-77

29. Abou-Khalil R, Yang F, Lieu S, Julien A, Perry J, Pereira C, Relaix F, Miclau T, Marcucio R, Colnot C (2015) Role of muscle stem cells during skeletal regeneration. Stem Cells 33:1501-1511

30. Liu R, Birke O, Morse A, Peacock L, Mikulec K, Little DG, Schindeler A (2011) Myogenic progenitors contribute to open but not closed fracture repair. BMC Musculoskelet Disord 12:288
31. Zannit HM, Silva MJ (2019) Proliferation and activation of osterix-lineage cells contribute to loading-induced periosteal bone formation in mice. JBMR Plus. https://doi.org/10.1002/ jbm4.10227

32. Park D, Spencer JA, Koh BI, Kobayashi T, Fujisaki J, Clemens TL, Lin CP, Kronenberg HM, Scadden DT (2012) Endogenous bone marrow MSCs are dynamic, fate-restricted participants in bone maintenance and regeneration. Cell Stem Cell 10:259-272

33. Moore ER, Zhu YX, Ryu HS, Jacobs CR (2018) Periosteal progenitors contribute to load-induced bone formation in adult mice and require primary cilia to sense mechanical stimulation. Stem Cell Res Ther 9:190

Publisher's Note Springer Nature remains neutral with regard to jurisdictional claims in published maps and institutional affiliations. 